\section{Dynamic Multiphoton Imaging: A Live View from Cells to Systems}

Grace E. Stutzmann and lan Parker University of California-Irvine, Irvine, California

Leaps in scientific technology often occur at the interface of seemingly disparate disciplines. This holds true with the recent application of multiphoton microscopy to the biological sciences, leading to a new generation of imaging-based studies extending from the tracking of individual molecules within living cells to the observation of whole organisms.

The theoretical concept of multiphoton excitation was first proposed by physicist Maria Göppert-Mayer in 1931 (11) but was not experimentally proven until the invention of the laser 30 years later, and even then it required decades of further development in laser technology to become of practical utility. The fields of physical chemistry and physics were the first to apply this technique $(12,33)$, and biological research has only more recently embraced multiphoton excitation to image living cells $(7,8)$. Biological uses of multiphoton microscopy have been extensively reviewed (e.g., Refs. 9 and 29). Here, we provide an introduction to its operating principles, discuss its advantages and limitations, and illustrate some recent applications that bring to light the strengths of this technology.

Fluorescence imaging is widely used in biology for morphological and functional studies, but conventional (singlephoton) fluorescence microscopy yields poor images, because in-focus structures are washed out by fluorescence outside the plane of focus. This can be mitigated by cutting thin sections (equal to or less than the width of a single cell), but that approach is clearly inapplicable for living tissue. The introduction of confocal microscopy thus represented an important advance, providing an "optical-sectioning" effect by using a pinhole aperture to reject out-of-focus fluorescence (41). Nevertheless, confocal microscopy still suffers from drawbacks, including limited (a few tens of micrometers) imaging depth into scattering biological tissues, photodamage owing to the use of short (high-energy) excitation wavelengths, and photobleaching. The subsequent development of multiphoton excitation greatly minimized these problems while sharing the optical-sectioning ability of confocal microscopy.

\section{How Does Multiphoton Microscopy Work?}

The principle of multiphoton excitation is elegantly simple. In the most commonly used case of two-photon microscopy, a fluorophore molecule is excited by the nearly simultaneous absorption (within $10^{-18} \mathrm{~s}$ ) of two photons, each about twice the wavelength (half the energy) required for singlephoton excitation. Likewise, threephoton excitation results from nearly simultaneous absorption of three photons, each having approximately onethird the energy needed for excitation of the fluorophore. The resulting fluorescent emission is then proportional to the square of the excitation intensity in two-photon absorption (or third power in the case of three-photon excitation). This nonlinear relationship provides the optical-sectioning ability of multiphoton imaging. Laser light focused on the specimen through a microscope objective has a high photon density in the focal point, but photon density, and therefore fluorescence excitation, falls rapidly away from this point. Fluorescence is generated only at the focal spot, and, unlike in confocal microscopy, there is essentially no out-of-focus light to reject (FIGURE 1A). A remaining problem is that multiphoton excitation requires enormously high light intensities that, if continuous, would almost instantly vaporize the specimen. The trick is to use lasers that produce amazingly brief $\left(\sim 10^{-13} \mathrm{~s}\right)$ pulses at a high repeti- tion rate (typically $\sim 80 \mathrm{MHz}$ ), thus generating very high instantaneous energy but low average energy (FIGURE 1B). Indeed, the adoption of multiphoton microscopy by biologists has largely followed progressive improvements in reliability and user friendliness of such "femtosecond" lasers.

In terms of optical-sectioning ability and image resolution, multiphoton microscopy has little advantage over confocal microscopy (6). However, several major advantages accrue secondarily from the use of long excitation wavelengths:

1) Light scattering declines steeply with increasing wavelength, so that infrared light (700-1,000 nm) used for two-photon imaging penetrates much deeper into tissue ( $500 \mu \mathrm{m})$ than the equivalent blue light (350-500 nm) used for one-photon excitation $(8,41)$.

2) Infrared light causes negligible photodamage or phototoxicity to cells. This is particularly advantageous for fluorophores and endogenous proteins that would normally require excitation at short $(<300 \mathrm{nM})$ and highly damaging ultraviolet wavelengths but can be made to fluoresce by three-photon excitation at infrared wavelengths three times longer $(18,44)$.

3) Photobleaching is restricted to the plane of focus.

4) The two-photon excitation spectra of most fluorophores are broader than for one-photon excitation, so a single multiphoton excitation wavelength can be used to simultaneously excite multiple fluorophores with distinct emission wavelengths. 
A Spatial compression of photons by objective lens

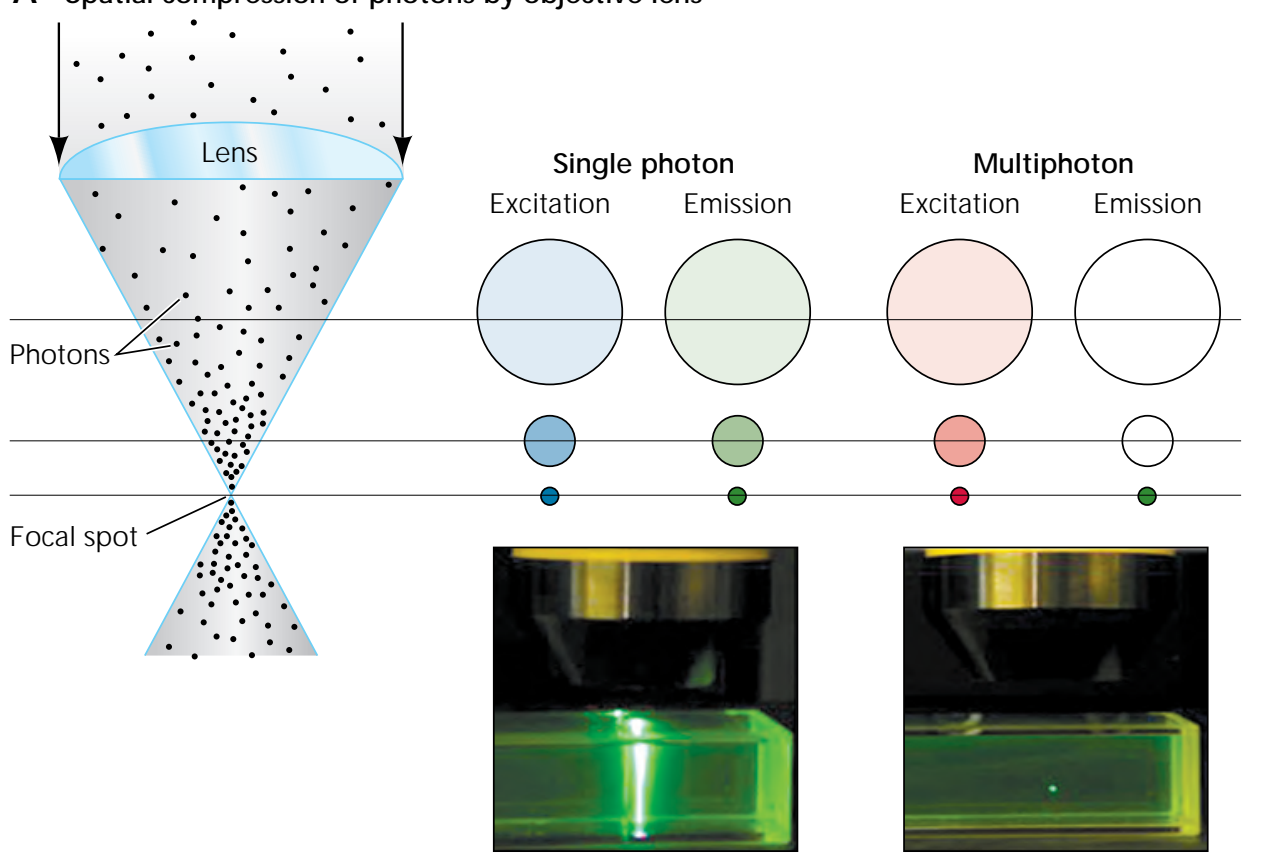

B Temporal compression of photons during femtosecond pulses

Continuous laser

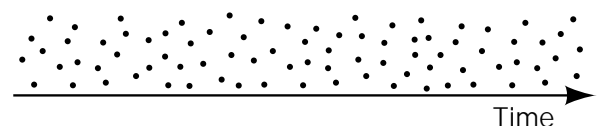

Femtosecond-pulsed laser

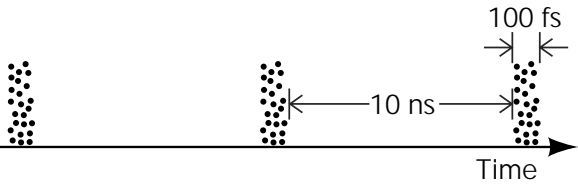

FIGURE 1. Principles and imaging modalities of multiphoton microscopy

A: demonstration of the differences between single-photon vs. multiphoton laser excitation. In both cases, the spatial compression of photons by an objective lens is greatest in the focal spot, and the photon density rapidly decreases away from this region. However, with conventional single-photon excitation, fluorophore excitation occurs throughout the beam path, resulting in fluorescence emission above and below the plane of focus. Multiphoton excitation restricts emission to the focal spot, owing to the quadratic relationship between excitation intensity and fluorescence emission. Photographs illustrate fluorescence in a cuvette of fluoresceine resulting from focused laser beams using single-photon excitation at $488 \mathrm{~nm}$ (left) and two-photon excitation with 780-nm, femtosecond pulses (right). Figure adapted from Ref. 4 with permission (see http://www.nature.com). $B$ : temporal compression of photons into $100-\mathrm{fs}$ packets achieves the high instantaneous power needed for multiphoton excitation, with an average power not much greater than a continuous laser beam as would be used for confocal imaging.

\section{Novel Imaging Modalities}

Multiphoton microscopy is most commonly used to acquire three-dimensional views of specimens labeled with a fluorescent probe but can also be used advantageously in conjunction with other microscopy techniques. One example is fluorescence correlation spectroscopy (FCS), in which fluctuations in fluorescence intensity of a small number of molecules within the focal spot are used to measure diffusion kinetics of individual molecules, protein-protein interactions, and molecular concentrations $(15,32)$. Applications of multiphoton FCS include analysis of aggregated $\beta$ amyloid peptides in human cerebrospinal fluid, multimerization of voltagesensitive $\mathrm{Ca}^{2+}$ channels, and alterations in signaling proteins after changes in neuronal plasticity (24). FCS provides a quantitative, molecular assay in live cells and represents a marked advancement over existing quantitative diffusion-based approaches such as fluorescence recovery after photo- bleaching (FRAP; Ref. 31). Another example is fluorescence lifetime imaging (FLIM), which measures changes in the lifetime of a fluorophore probe that depend on its local environment (e.g., pH) but are independent of concentration. For example, although DNA and RNA molecules have a similar affinity for nucleic acid stains, they can readily be distinguished in living and apoptotic cells by virtue of differences in fluorescence lifetimes of certain nucleic acid dyes when bound to DNA compared with RNA (39).

FCS and FLIM are both possible using conventional (single-photon) microscopy but benefit from the increased depth penetration and minimal phototoxicity of multiphoton imaging. Beyond this, the femtosecond laser pulses required for multiphoton microscopy enable imaging techniques that are not possible by other means. Thus second harmonic generation (SHG) microscopy uses nonlinear optical properties that emerge at the very high energies during focused laser pulses. However, rather than being based on absorption of light energy by fluorescent molecules, SHG uses inherent properties of highly ordered structures that inherently function as frequency doublers of the excitation light. For example, infrared $(900 \mathrm{~nm})$ femtosecond pulses result in deep blue light at exactly one-half the wavelength $(450 \mathrm{~nm})$ traveling in the same direction as the incident light. SHG is well suited for imaging of ordered structures (e.g., collagen and myosin) deep within tissue without the need for any exogenous probes (5), provides information about molecular orientation and symmetries (27), and can be applied simultaneously with multiphoton fluorescence imaging to provide additional information about the specific molecular source of the SHG $(27,45)$. Recent developments of functional SHG probes, such as membrane voltage sensors with uniquely high sensitivity (30), hold further promise. 
Finally, we note that multiphoton microscopy is not limited merely to imaging but can be applied to perturb biological tissues with very high spatial and temporal specificity. Examples include the localized "uncaging" of biologically active compounds such as neurotransmitters or intracellular messengers from photolabile precursors to reveal inter- and intracellular ionic communication (34); the three-photon induction of a $\mathrm{red} / \mathrm{green}$ color change of a red fluorescent protein (DsRed) as a way of optically "highlighting" individual cells or subcellular regions (FIGURE 2A; Ref. 21); and the selective ablation of individual organelles, and even

\section{A DsRed-expressing HEK cells}
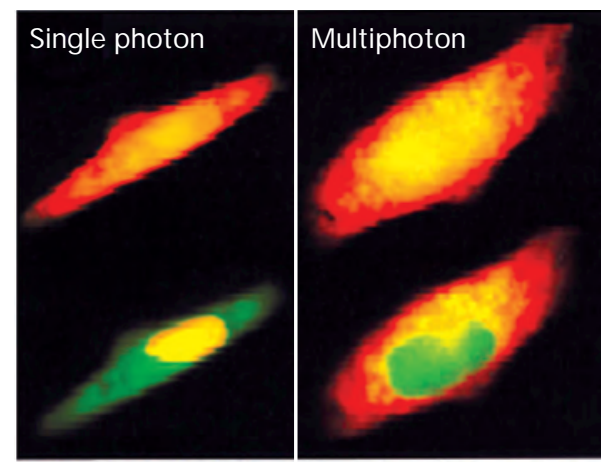

C $\mathrm{Ca}^{2+}$-dependent fluorescence
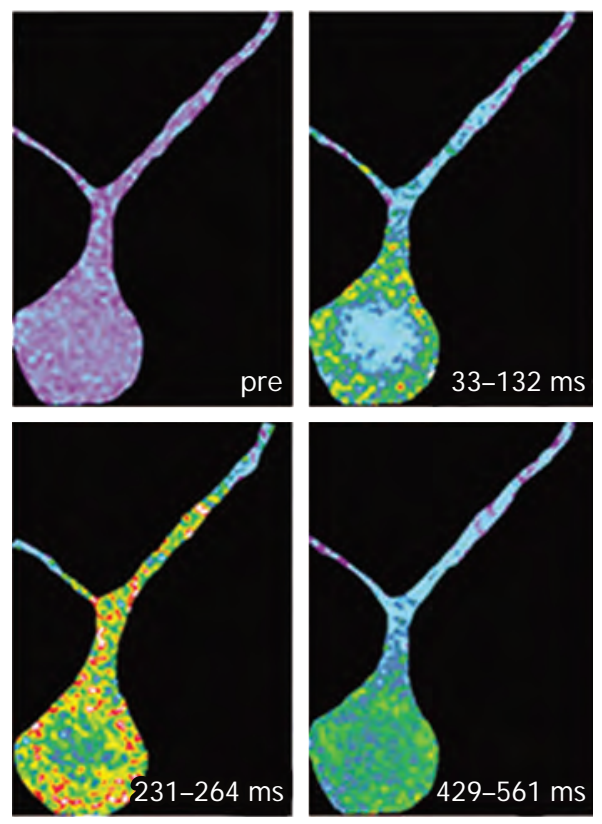

the use of the femtosecond laser beam as an optical microtome to construct three-dimensional images of entire fixed organs or organisms by sequentially imaging sections some tens of micrometers thick and then (by turning up the laser power) ablating that section to expose fresh tissue underneath $(1,38)$.

\section{Biological Applications}

Multiphoton imaging is not a panacea for all microscopy problems, and for many purposes confocal microscopy may be preferable (and less expensive), such as in protocols in which rapid exchange between ex- citation wavelengths is needed (e.g., ratiometric imaging). However, its inherent optical sectioning, deeper penetration in scattering tissue, and reduced phototoxicity are particularly applicable to dynamic imaging of living specimens. Here, we illustrate applications at levels from the subcellular to entire organisms that exemplify the advantages of multiphoton microscopy.

\section{Subcellular/organelles}

Neurobiologists were among the first to harness multiphoton microscopy for biological studies. A key functional component in the nervous system is the dendritic spine, a tiny structure

B Dendritic arborization in mouse barrel cortex

Day 2

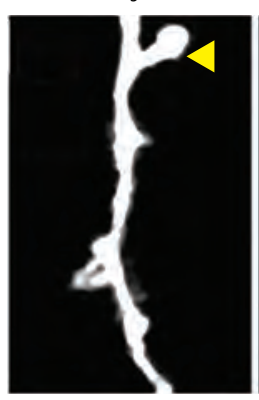

D $\mathrm{Ca}^{2+}$ transients in apical dendrites

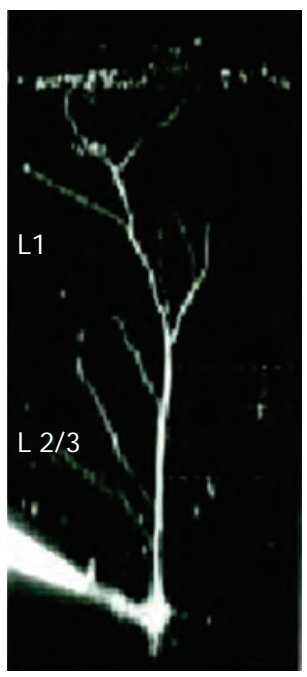

Day 3

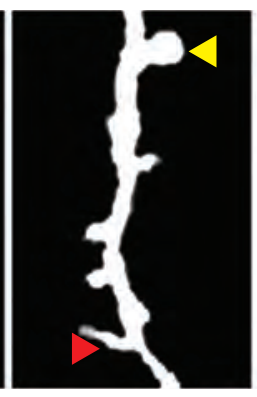

$50 \mu \mathrm{M}$

$20 \%$ $1 \mathrm{~s}$

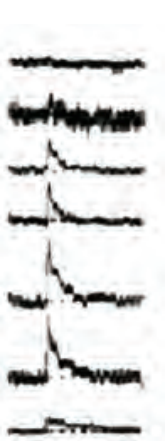

Day 4

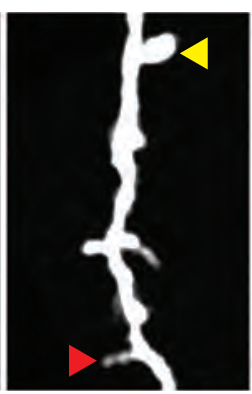

Day 5

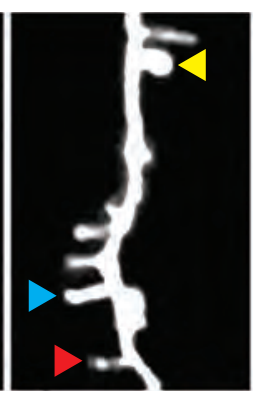

Day 6

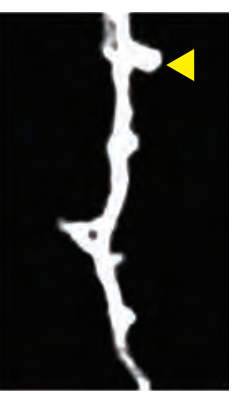

FIGURE 2. Multiphoton applications for in vivo and in vitro cellular and subcellular imaging

$A$ : application of the multiphoton "greening techA: application of the multiphoton "greening tech-
nique" for labeling subcellular regions. Image pairs show DsRed-expressing human embryonic kidney (HEK) cells imaged by conventional (single-photon) epifluorescence microscopy, before (upper) and after (lower) greening the cytoplasm (left) or the nucleus (right) by local exposure to focused, 760-nm, fem(right by local exposur. The focused, $760-n m^{\prime} f$ arises from a selective three-photon bleaching of the mature, red form of DsRed, resulting in reduced red fluorescence and (via a reduction in Forster resonance energy transfer) enhanced fluorescence of the immature green species. Figure adapted from Ref. 21 with permission (see http://www.nature.com). B: Longterm two-photon imaging of the same dendritic arborization within the barrel cortex of a mouse. arborization within the barrel cortex of a mouse. changed morphology (yellow) or appeared and retracted (blue, red) throughout an 8-day period. Figure adapted from Ref. 37 with permission (see http://www.nature.com). C: subcellular distribution of $\mathrm{Ca}^{2+}$-dependent fluorescence in a fura 2-loaded pyramidal neuron in an in vitro slice preparation imaged at different times following photorelease of the intracellular messenger inositol 1,4,5-trisphosphate. Figure adapted from Ref. 36 with permission. $D: \mathrm{Ca}^{2+}$ transients elicited by single action potentials along apical dendrites of a neuron visualized $400 \mu \mathrm{m}$ into the brain of a living rat using two-photon microscopy. The image shows a side view of resting fluorescence in a neuron loaded with a fluorescent $\mathrm{Ca}^{2+}$ indicator, reconstructed from an image stack acquired at increasing focal distances. Traces show changes in $\mathrm{Ca}^{2+}$

dependent fluorescence recorded at adjacent depths in image in response to single action potentials. Figure adapted from Ref. 40 with permission. 
about a micrometer in size that forms the postsynaptic element mediating communication between neurons. Much interest focuses on the chemical and electrical signaling mechanisms in spines and on the plastic changes in their distribution, morphology, and function that may underlie learning and memory. However, it has been difficult to image these dynamic processes in live preparations. Neurons are sensitive to photodamage, brain tissue is highly scattering, regions of interest may lie hundreds of micrometers below the surface of the intact brain, and although it is possible to maintain viable slices of brain, these must be relatively thick to preserve functional connections. The introduction of multiphoton microscopy thus offered enormous advantages in terms of high-resolution, deep, and noninjurious imaging. Recent examples of multiphoton imaging in 300- to 400$\mu \mathrm{m}$-thick brain slices demonstrate the morphology, unique signaling dynamics, and rapid motility of dendritic spine head and neck regions $(14,19)$. Most spectacularly, it has been possible to resolve individual dendrites and spines in living animals by creating a viewing window into the brain by removing or thinning the skull of mice expressing a green fluorescent protein in a small subpopulation of cortical neurons (37). These could then be imaged repeatedly over several weeks, revealing that spines appear and disappear frequently in the adult cortex and suggesting that these changes might underlie adaptive remodeling of neural circuits in response to sensory experience (FIGURE 2B).

\section{Dynamic cellular imaging}

In addition to morphological studies, multiphoton imaging of cellular activity can be accomplished with the use of various fluorescent probes. For example, $\mathrm{Ca}^{2+}$-indicator dyes can provide detailed spatial and temporal information about $\mathrm{Ca}^{2+}$ entry through voltage-gated channels in excitable cells or patterns of $\mathrm{Ca}^{2+}$ release from intracellular stores. Although visualizing cellular activities, such as ion flux or membrane voltage changes, can be accomplished by conventional fluorescence techniques, multiphoton excitation facilitates functional imaging of individual cells in thick tissue (FIGURE 2C; Ref. 34) and in intact animals (FIGURE 2D; Ref. 40) Yet a further advantage is nicely illustrated in recent studies using multiphoton excitation in retinal starburst amacrine cells that are involved in the processing of visual information yet are not activated by infrared wavelengths outside of the visible spectrum (10). Visible wavelengths were used to evoke patterned stimulation at the retinal photoreceptors, whereas multiphoton excitation (using infrared light) was used concurrently to excite a fluorescent $\mathrm{Ca}^{2+}$ indicator loaded into amacrine cells, revealing the functional responses to the patterned stimulation without complications due to photostimulation by the excitation light itself.

\section{Imaging within intact organs}

For many years biological research has pursued a reductionistic trend with an increasing focus on genomics and proteomics. Multiphoton microscopy now offers an important tool toward a more integrative approach, because its ability to image function and morphology at cellular, and even molecular levels deep within intact organs and living animals provides a means to unify molecular, cellular and organismal studies. This approach has far-reaching applications for understanding how multiple cells and cell types interact to determine the function of an entire organ and can provide insight into disease mechanisms and aid development of therapeutic strategies. An example is the application of multiphoton imaging to open a new window onto the immune system (FIGURE 3A; see Ref. 4 for a review). Activation of the immune response depends on a complex choreography of interactions between different cell types, yet despite a wealth of molecular knowledge, little is known of the cellcell interactions deep within native lymphoid tissue. Again, the depth penetration and minimal photodamage of multiphoton microscopy are crucial and, in conjunction with use of established fluorescent celltracker dyes to label adoptively transferred lymphocytes, permit sixdimensional imaging $(x, y, z$, time, intensity, and emission wavelength) into both excised lymph nodes and nodes in an anesthetized mouse. Time-lapse movies reveal strikingly dynamic movements and cellular interactions within the in vivo setting, in marked contrast to the static view of lymphocytes in culture, and are beginning to provide mechanistic insights into the processes by which $T$ cells search for antigen and subsequently become activated by repeated contacts with antigenpresenting cells $(2,23,26)$. The advantages of multiphoton imaging are further highlighted because individual naïve $T$ cells can be followed within the lymph node for hours without loss of viability or motility $(25,26)$, whereas analogous studies using (single-photon) confocal imaging concluded that these cells were essentially immotile (35), a result that may reflect photodamage by the shorter-wavelength excitation.

\section{Imaging whole organisms/embryos}

At even larger scales, multiphoton microscopes can be "zoomed out" to capture an entire embryo during development while maintaining high (subcellular) spatial and temporal resolution over long periods (days to weeks). Transparent organisms such as Caenorhabditis elegens, zebrafish, and Drosophila are ideal candidates for such studies, and "in toto" imaging of entire embryos has revealed expression patterns at the cellular and genomic levels (FIGURE 3B; Ref. 22). Multiphoton imaging enables longterm observation of biological activity by providing the ability to repeatedly track and monitor cells deep within a growing embryo or organism while minimizing photodamage to cell structure and DNA. 


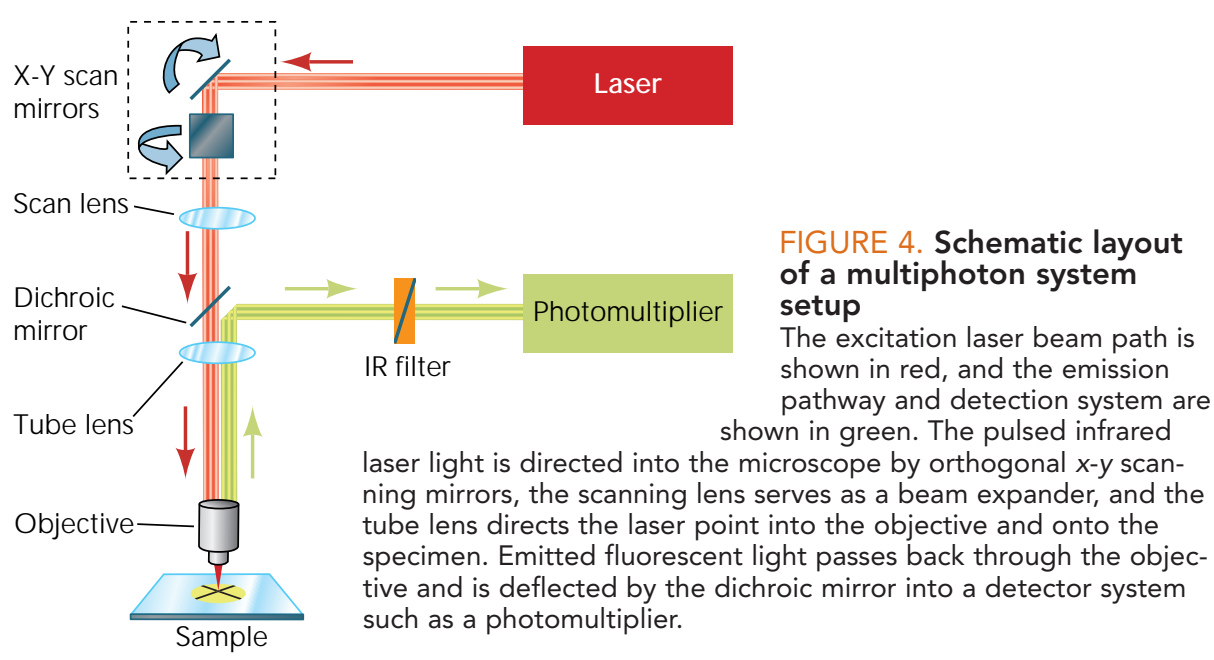

cellular activity from awake, behaving animals (see Ref. 13 for further reading).

On the receiving end of multiphoton excitation, quantum dots have great promise as an extremely bright alternative to conventional fluorophores. These are nanocrystals of semiconductor that have a very high two-photon cross-section, show minimal photobleaching, and are available in a range of emission wavelengths. Initial problems with biocompatibility appear now to be largely solved, and quantum dots are available coated with various substrates, allowing them, for example, to be conjugated to specific antibodies. (43)

\section{Applications}

The key strengths of multiphoton microscopy lie in its applications for real-time imaging of dynamic processes occurring within the native environment of intact live tissues, organs, and organisms. Developmental biologists and neurobiologists were among the first to use this technique, and its more recent adoption by immunologists and molecular biologists indicates its potential within other disciplines. Future applications for multiphoton microscopy are broad and exciting, and they extend beyond basic research into the realm of noninvasive or endoscopic clinical and diagnostic work, such as detection of skin cancers, Alzheimer's disease, and metabolic disorders just by "looking" into the patient with a cellular level of resolution (45).

\section{Appendix: Constructing Your Own Multiphoton System}

Technological limitations of earlier generations of femtosecond lasers were originally a great deterrent to their use by biologists, but current models are compact and require no manual intervention by the user However, the considerable expense of commercial multiphoton systems $(\sim 700,000)$ remains a hurdle. Because of this, many multiphoton microscopes are purchased for multiuser facilities-a job for which they are often ill suited-and end up gathering dust! Instead, multiphoton microscopes are better employed within the laboratory of an individual investigator, where they can be dedicated for extended experiments and equipped as needed with ancillary systems for superfusion environmental control, electrophysiological recording, etc. Several of the applications described in this review were made possible because the investigators constructed their own multiphoton microscopes at a considerable saving in cost $(17,20,28)$ This task is not as daunting as might be feared and, beyond the intimate working knowledge and inherent satisfaction it provides, has further advantages, such as that the system can be customized for specific applications. FIGURE 4 outlines the basic working pathway and components in a multiphoton imaging system, and the references provide detailed instructions for constructing one's own system.

We thank Dr. Mark Miller for helpful discussions.

Work in the authors' laboratory is supported by grant GM-48071 from the National Institute of General Medical Sciences.

\section{References}

1. Arrigoni M. Femtosecond laser pulses in biology: from microscopy to ablation and micromanipulation. Biophotonics Int: 48-51, 2004

2. Bousso $P$ and Robey E. Dynamics of $C D 8^{+} T$ cell priming by dendritic cells in intact lymph nodes. Nat Immun 4: 579-585, 2003.

3. Buist $A H$, Muller M, Squier J, and Brakenhoff GJ. Real time two-photon absorption microscopy using multipoint excitation. J Microsc 192 217-226, 1998

4. Cahalan MD, Parker I, Wei SH, and Miller M. Twophoton tissue imaging: seeing the immune system in a fresh light. Nat Rev Immunol 2: 872-800, 2002.

5. Campagnola PJ, Mohler W, and Millard AE. Three-dimensional high-resolution second-harmonic gen tion haging of endogenous struc493-508, 2002.

6. Cox $G$ and Sheppard CJR. Practical limits of resolution in confocal and non-linear microscopy. Microsc Res Tech 63: 18-22, 2004.

7. Denk W, Delaney KR, Gelperin A, Kleinfeld D, Strowbridge BW, Tank DW, and Yuste R. Anatomical and functional imaging of neurons using 2-photon laser scanning microscopy. J

8. Denk W, Strickler J, and Webb W. Two-photon laser scanning fluorescence microscopy. Science 248: 73-76, 1990

9. Diaspro Ed A. Two-photon microscopy-Part II. Microsc Res Tech 63: 1-86, 2004.

10. Euler T, Detwiler PB, and Denk W. Directionally elective calcium signals in dendrites of starburst selective calcium signals in dendrites of starbu
amacrine cells. Nature 418: 845-852, 2002.

11. Göppert-Mayer M. Über Elementarakte mit zwei Quantensprüngen. Ann Phys (Leipzig) 9: 273-294 1931

12. Hellwarth $R$ and Christensen P. Nonlinear optical Optination of structures in polycrysallin Znse. Opt Commun 12. 318 322, 1974.

13. Helmchen F. Miniaturization of fluorescence microscopes using fibre optics. Exp Physiol 87: 737-745, 2002.

14. Holthoff K, Tsay D, and Yuste R. Calcium dynamics of spines depend on their dendritic location. Neuron 33: 425-437, 2002.

15. Kim S and Schwille P. Intracellular applications of fluorescence correlation spectroscopy: prospects or neuroscienc

16. Levene MJ, Dombeck DA, Kasischke KA, Molloy $\mathrm{RP}$, and Webb WW. In vivo multiphoton microscopy of deep brain tissue. J Neurophysiol 91: 1908-1912, 2004.

17. Mainen $Z$, Maletic-Savatic M, Shi S, Hayashi $Y$, Malinow R, and Svoboda K. Two-photon imaging in living brain slices. Methods 18: 231-239, 1999. 


\section{(A3 I I II}

18. Maiti S, Shear J, Williams R, Zipfel W, and Webb $W$. Measuring serotonin distribution in live cells with three photon excitation. Science 275: 530-532, 1997

19. Majewska A, Tashiro A, and Yuste R. Regulation of spine calcium dynamics by rapid spine motility. Neurosci Methods 20: 8262-8268, 2000.

20. Majewska A, Yiu G, and Yuste R. A custom-made two-photon microscope and deconvolution system. Pflügers Arch 441: 398-408, 2000.

21. Marchant J, Stutzmann G, Leissring M, LaFerla F and Parker I. Multiphoton-evoked color change of DsRed as an optical highlighter for cellular and subcellular labeling. Nat Biotechnol 19: 654-659, 2001.

22. Megason SG and Fraser S. Digitizing life at the level of the cell: high-performance laser-scanning microscopy and image analysis for in toto imag2003.

23. Mempel TR, Henrickson SE, and Von Andrian U. Tcell priming by dendritic cells in lymph nodes occurs in three distinct phases. Nature 8 154-159, 2004

24. Meyer $T$ and Shen K. In and out of the postsynaptic region: signalling proteins on the move. Trends Cell Biol 10: 238-244, 2000.

25. Miller MJ, Wei SH, Cahalan MD, and Parker I. Autonomous $\mathrm{T}$ cell trafficking examined in vivo with intravital two-photon microscopy. Proc Nat Acad Sci USA 100: 2604-2609, 2003.

26. Miller MJ, Wei SH, Parker I, and Cahalan M. Two photon imaging of lymphocyte motility and antigen response in intact lymph node.

27. Mohler W, Millard A, and Campagnola P. Second harmonic generation imaging of endogenous
structural proteins. Methods 29: 97-109, 2003.
28. Nguyen $Q$, Callamaras $N$, and Parker I. Construction of a two-photon microscope for
video-rate $\mathrm{Ca}^{2+}$ imaging. Cell Calcium 30: 383-393, 2001.

29. Niggli E and Egger M. Applications of multi-photon microscopy in cell physiology. Front Biosci 1 :

30. Pons $T$, Moreaux L, Mongin O, Blanchard-Desce $\mathrm{M}$, and Mertz J. Mechanisms of membrane potential sensing with second-harmonic genera-
tion microscopy. J Biomed Opt 8: 428-431, 2003.

31. Reits EA and Neefjes J. From fixed to FRAP: measuring protein mobility and activity in living cells. Nat Cell Biol 3: E145-E147, 2001.

32. Schwille $P$, Haupts $U$, Maiti $S$, and Webb W Molecular dynamics in living cells observed by fluorescence correlation spectroscopy with one-and two-photon excitation. Biophys J 77: 2251-2265, 1999.

33. Sheppard C and Kompfner R. Resonant scanning optical microscope. Appl Opt 17: 2879-2882, 1978.

34. Soeller C, Jacobs MD, Jones KT, Ellis-Davies GC Donaldson PJ, and Cannell M. Application of two-photon flash photolysis to reveal intercellular ments. J Biomed Opt 8: 418-427, 2003.

35. Stoll S, Delon J, Brotz TM, and Germain R. Dynamic imaging of $T$ cell-dendritic cell interactions in lymph nodes. Science 296: 1873-1876, 2002.

36. Stutzmann GE, LaFerla FM, and Parker I. $\mathrm{Ca}^{2+}$ signaling in mouse cortical neurons studied by twophoton imaging phiphos imate. J Neurosci 23: 758-765, 2003.
37. Trachtenberg JT, Chen BE, Knott GW, Feng G, Sanes JR, Welker E, and Svoboda K. Long-term in vivo imaging of experience-dependent synaptic
plasticity in adult cortex. Nature 420: 788-794, 2002.

38. Tsai PS, Friedman $B$, Ifarraguerri Al, Thompson BD, Lev-Ram V, Schaffer CB, Xiong O, Tsien RY, Squier $\mathrm{JA}$, and Kleinfeld D. All-optical histology 2003.

39. Van Zandvoort M, de Grauw C, Gerritsen H, Broers J, oude Egbrink M, Ramaekers F, and Slaaf vital fluorescent probe: lifetime imaging of SYTO13 in healthy and apoptotic cells. Cytometry 47: 226-235, 2002.

40. Waters J, Larkum M, Sakmann B, and Helmchen F. Supralinear $\mathrm{Ca}^{2+}$ influx into dendritic tufts of layer 2/3 neocortical pyramidal neurons in

41. Webb R. Theoretical basis of confocal microscopy. Methods Enzymol 307: 3-20, 1999.

42. Williams RM, Zipfel WR, and Webb W. Multiphoton microscopy in biological research. Curr Opin Chem Biol 5: 603-608, 2001.

43. Wu X, Liu H, Liu J, Haley KN, Treadway JA, Larson $\mathrm{JP}, \quad \mathrm{Ge} \mathrm{N}_{\text {, Peale }} \mathrm{F}_{1}$ and Bruchez MP. Her2 and other cellular targets with semiconductor quantum dots. Nat Biotechnol 21: 41-46, 2003

44. Xu C, Zipfel W, Shear JB, Williams RM, and Webb W. Multiphoton fluorescence excitation: new spectral windows for biological nonlinear microscopy. Proc Natl Acad Sci USA 93: 10763-10768,

45. Zipfel WR, Williams RM, Christie R, Nikitin AY, Hyman BT, and Webb W. Live tissue intrinsic Hyman $B T$, and Webb $W$. Live tissue intrinsic native fluores ation. Proc Natl Acad Sci USA 100: 7075-7080, 2003. 